Nervenarzt 2011 $\cdot 82: 143-144$ DOI 10.1007/s00115-010-3109-2

Online publiziert: 2. Februar 2011

(c) Springer-Verlag 2011

\author{
J. Röther ${ }^{1} \cdot$ U. Laufs ${ }^{2}$ \\ ${ }^{1}$ Asklepios Klinik Altona, Hamburg \\ ${ }^{2}$ Klinik für Innere Medizin III, Kardiologie, Angiologie und Internistische \\ Intensivmedizin, Universitätsklinikum des Saarlandes, Homburg/Saar
}

\title{
Schnittstelle Herz und Hirn
}

\section{Herausforderungen und Chancen}

Herz und Hirn stehen in vielfältigen Beziehungen zueinander: Vaskuläre Erkrankungen von Herz und Gehirn werden durch die gleichen Risikofaktoren bedingt und weisen Parallelen hinsichtlich ihrer Pathogenese auf. Patienten mit zerebraler Ischämie haben ein hohes Herzinfarktrisiko und profitieren im Rahmen der Sekundärprävention durch eine Reduktion der Ereignisse in beiden Stromgebieten [1, $2,3]$. Umgekehrt haben Patienten mit koronarer Herzkrankheit und Patienten mit Herzinsuffizienz ein erhöhtes Schlaganfallrisiko $[1,2,3]$.

Das Herz ist eine wichtige Emboliequelle. Eine besondere Bedeutung kommt dem Vorhofflimmern zu, welches jedem 4. Schlaganfall zugrunde liegt. Neue Entwicklungen hinsichtlich der Antikoagulation, der antiarrhythmischen Therapie sowie interventionelle Techniken werden das therapeutische Spektrum in den nächsten Jahren wesentlich erweitern und sind echte „Herz-und-Hirn-Themen“, erfordern sie doch eine enge Zusammenarbeit zwischen Kardiologen und Neurologen [4].

\section{( ) Neue Strategien erfordern die enge Zusammenarbeit von Kardiologen und Neurologen}

All dies war Motivation für das vorliegende Leitthemenheft „Herz und Hirn“. Im ersten Beitrag werden epidemiologische Faktoren der koronaren Herzkrankheit und des Schlaganfalls gegenübergestellt. Für beide Erkrankungen trifft $\mathrm{zu}$, dass aufgrund der erfolgreichen Sekundärprävention die Mortalität gesunken ist. Aufgrund der steigenden Lebens- erwartungen und der damit verbundenen größeren Zahl älterer Menschen werden jedoch die absoluten Zahlen von HerzKreislauf-Erkrankungen und Schlaganfall in der EU von 1,1 Mio. Betroffenen im Jahr 2000 auf 1,5 Mio. im Jahr 2025 ansteigen. Dies bedeutet gleichzeitig eine Zunahme älterer Menschen mit Mehrfachmedikation. Allerdings liegt der Anteil der Patienten nach Schlaganfall und kardiovaskulären Erkrankungen, die die zur Sekundärprävention verordneten Medikamente nicht dauerhaft einnehmen, bei $20-50 \%$. Strategien mit dem Ziel der Verbesserung der Einnahmetreue könnten daher einen hohen Nutzen sowohl für den Patienten als auch hinsichtlich der Behandlungskosten erbringen. Dies wird jedoch nur durch umfangreiche Maßnahmen wie eine individuelle Patientenberatung, Medikationsmanagement, z. B. mithilfe von Wochenblistern, und eine patientenbezogene individualisierte Therapie möglich sein, deren Wirksamkeit allerdings überprüft werden muss.

Der Detektion von Vorhofflimmern kommt eine große Bedeutung zu. Bei jedem 20. Patienten führt der Schlaganfall zur Erstdiagnose von Vorhofflimmern. Vorhofflimmern rechtzeitig zu erkennen, ist daher Ziel der Prävention. Ein besserer Nachweis während des Monitorings auf der Stroke-Unit, eine Verlängerung der Holter-EKG-Aufzeichnungen von $24 \mathrm{~h}$ auf 7 Tage sowie der Einsatz implantierbarer Ereignisrekorder für ausgewählte Patienten können die Detektion von bisher unbekanntem Vorhofflimmern wesentlich erhöhen.

Eine potenzielle neue Möglichkeit, das Risiko kardialer Embolien bei Vor- hofflimmern zu minimieren, ist der interventionelle Verschluss des Vorhofohrs. Auch wenn die Studienlage zu dieser noch jungen interventionellen Technik nicht befriedigend ist, so kann die Indikation bei Patienten mit erhöhtem Blutungsrisiko unter Antikoagulation und gleichzeitigem hohem Embolierisiko bei Vorhofflimmern in Einzelfällen gestellt werden. Auch in Bezug auf die pharmakologische Prävention von Schlaganfällen bei Vorhofflimmern gibt es vielversprechende neue Präparate. Die Entwicklung spezifisch an den zentralen Gerinnungsfaktoren Thrombin und Faktor Xa ansetzender oraler Inhibitoren ermöglicht eine zuverlässige Antikoagulation ohne regelmäßiges Gerinnungsmonitoring. Jetzt stehen belastbare klinische Studiendaten zur Verfügung, um zu entscheiden, welche Patienten von den neuen Thrombin- und Faktor-Xa-Hemmern profitieren können.

Im letzten Jahr hat die Arbeitsgemeinschaft Herz und Hirn der Deutschen Gesellschaft für Kardiologie und der Deutschen Schlaganfall-Gesellschaft zu der Bedeutung und den Behandlungsmöglichkeiten einer zerebralen Ischämie bei Herzkatheteruntersuchungen in einem Konsensuspapier Stellung genommen [3]. Allerdings ist die neurologische Expertise auch bei herzchirurgischen Eingriffen gefragt. Der letzte Beitrag in diesem Themenheft referiert daher die kognitive Beeinträchtigung nach kardiochi-

Joachim Röther und Ulrich Laufs: Arbeitsgemeinschaft Herz und Hirn der Deutschen Gesellschaft für Kardiologie und der Deutschen Schlaganfall-Gesellschaft. 
rurgischen Eingriffen, bei der Mikroembolisation und vorbestehende zerebrovaskuläre Risikofaktoren eine wesentliche Rolle spielen, und diskutiert präventive Strategien.

Als Sprecher der Arbeitsgemeinschaft Herz und Hirn freuen wir uns, Ihnen einen aktuellen Überblick über wichtige Herz- und Hirnthemen geben zu können. Wir bedanken uns bei den Autoren für die Beiträge und setzen auf weiterhin gute Zusammenarbeit.

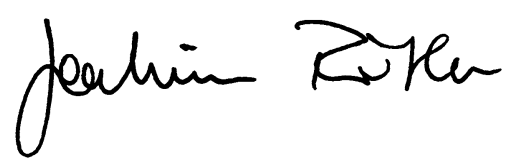

Prof. Dr. Joachim Röther

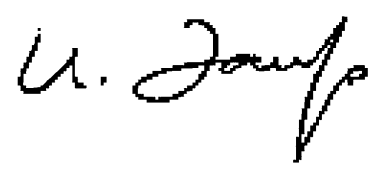

Prof. Dr. Ulrich Laufs

\section{Korrespondenzadresse}

\section{Prof. Dr. J. Röther}

Asklepios Klinik Altona

Paul-Ehrlich-Straße 1, 22763 Hamburg

j.roether@asklepios.com

\section{Prof. Dr. U. Laufs}

Klinik für Innere Medizin III, Kardiologie, Angiologie und Internistische Intensivmedizin, Universitätsklinikum des Saarlandes $66421 \mathrm{Homburg} / \mathrm{Saar}$

ulrich@laufs.com

\section{Literatur}

1. Laufs U, Hoppe UC, Rosenkranz S et al (2010) Cardiological evaluation after cerebral ischaemia: consensus statement of the Working Group Heart and Brain of the German Cardiac Society-Cardiovascular Research (DGK) and the German Stroke Society (DSG). Clin Res Cardiol 99(10):609-625

2. Laufs U, Hoppe UC, Rosenkranz S et al (2010) Cardiac workup after cerebral ischemia: consensus paper of the Working Group on Heart and Brain of the German Cardiac Society and German Stroke Society. Nervenarzt 81(4):444-462

3. Röther J, Laufs U, Böhm M et al (2009) Consensus paper on peri-interventional and postinterventional stroke during cardiac catheter procedures. Nervenarzt 80(10):1205-1206, 1208-12015

4. Röther J, Laufs U (2010) Preventing stroke by treating atrial fibrillation - new hope with dronedarone and dabigatran? Dtsch Med Wochenschr 135 (Suppl 2):55-58

\section{Dem Schwindel auf der Spur} Eröffnung der IFB ${ }^{\mathrm{LMU}}$ Schwindelambulanz in München

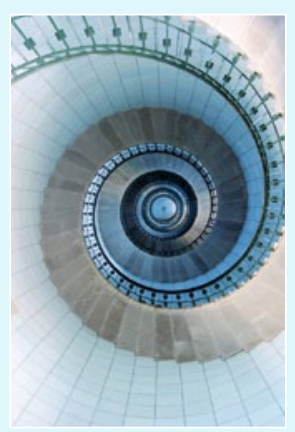

Am Integrierten Forschungs- und Behandlungszentrum für Schwindel, Gleichgewichts- und Augenbewegungsstörungen IFB ${ }^{\mathrm{LMU}}$ in München (Direktor: Prof. Thomas Brandt)

wurde am 3. Februar 2011 die neue Schwindelambulanz eröffnet.

Die Schwindelambulanz ist eine weltweit einzigartige interdisziplinäre Anlaufstelle für Patienten mit Schwindelsymptomen. Keine andere Schwindelambulanz vereint so viele Disziplinen (Neurologie, HNO, Ophthalmologie, Psychosomatik, Radiologie, Neuroradiologie, Nuklearmedizin, Innere Medizin, Neuropädiatrie) unter einem Dach und bietet dadurch Hilfe auch für Personen mit schweren oder ungewöhnlichen Schwindelformen. Die Behandlung der Patienten erfolgt hier nach gemeinsamen Leitlinien zu Diagnose und Therapie.

Die neu eröffnete Schwindelambulanz bündelt bereits bestehende Ambulanzen personell und räumlich an einem Ort und bildet eine Einheit in der von der Erstdiagnostik über die Therapie bis hin zu Verlaufskontrollen eine umfassende Betreuung von Schwindelpatienten erfolgen kann. Jährlich sollen hier etwa 3.000 Patienten behandelt werden.

Im Anschluss an die Eröffnung der Schwindelambulanz gaben Expertinnen und Experten auf der Fachkonferenz "Forschung konkret: Dem Schwindel auf der Spur" Einblicke in die Arbeit und Forschungsschwerpunkte des Integrierten Forschungs- und Behandlungszentrums $\mathrm{IFB}^{\mathrm{LMU}}$ für Schwindel, Gleichgewichts- und Augenbewegungsstörungen.

Mit den „Integrierten Forschungs- und Behandlungszentren (IFB)" fördert das Bundesministerium für Bildung und Forschung (BMBF) die enge Verknüpfung von Forschung und Patientenversorgung. Mit einem IFB soll in einem bedeutsamen Krankheitsbereich der nachhaltige Aufbau eines Forschung und Versorgung umfassenden Zentrums zu einem gemeinsamen Schwerpunkt gestärkt werden. Durch die Überwindung traditioneller Fachgrenzen kann in einem IFB interdisziplinär auf hohem Niveau gearbeitet werden. Patienten sollen künftig noch schneller von neuen Forschungsergebnissen profitieren. Ziel ist auch die Verbesserung der Nachwuchsförderung, um hochqualifizierte Expertinnen und Experten in Deutschland zu fördern und zu binden und den Forschungsstandort Deutschland zu stärken.

Quelle: Bundesministerium für Bildung und Forschung (www.bmbf.de) 